\title{
Occlusal pitfalls and how to avoid them
}

\author{
R. Wassell ${ }^{1}$
}

Occlusion conjures up various emotions in dentists. Some say they were taught nothing about it at dental school so it must be unimportant. Others, through their own experience and postgraduate study, realise that misdirected or excessive occlusal forces can be damaging to restorations, teeth/implants and periodontium. However, they shy away from actively managing patients' occlusions because they fear retribution from their patients or colleagues. A third group actively embraces all things occlusion, but sadly uncritical enthusiasm may lead to inappropriate or excessive treatment. In my lecture I aim to raise awareness of occlusal pitfalls and suggest ways towards a more predictable treatment outcome.

While some occlusal problems occur naturally many are iatrogenic. These often occur while providing restorations or carrying out occlusal adjustment and splint treatment. They result simply from a failure to ensure adequate occlusal harmony and occlusal stability. In bruxists occlusal problems are aggravated by the heavy occlusal loading. However, it is not all down to biomechanics as some patients become 'hypervigilent' about the way their teeth meet, find it difficult to adapt to occlusal changes and can be notoriously challenging to treat.

\section{Occlusal pitfalls with restorations}

To avoid occlusal pitfalls with restorations means going back to basics, being selfcritical about your dentistry and not just blaming the technician. Remember a high crown is the end result of a chain of procedures involving occlusal examination, tooth preparation, impressions, jaw registration and cast mounting. Any one of these may incorporate an error including the humble alginate impression used to record the opposing arch.

${ }^{1}$ Consultant and Senior Lecturer in Restorative Dentistry (Conservation), School of Dental Sciences, Newcastle University, Newcastle upon Tyne, NE2 4BW

Correspondence to: Robert Wassell

Email: robert.wassell@ncl.ac.uk

DOI: 10.1038/sj.bdj.2012.255
You could, of course, fit a crown high and hope the patient will 'bite-it-in'. Indeed this is a respectable approach if the crown is used as a 'Dahl Appliance', but why risk disrupting a patient's occlusion if you don't need orthodontic movement to create occlusal space? Although patients may only suffer discomfort for a week or two, more seriously in susceptible individuals it may precipitate temporomandibular disorders (TMD) or facial pain. The risk appears greater if the occlusal loading on the high restoration is non-axial with the additional risk of biomechanical overload.

Dentists may precipitate a variety of biomechanical problems with anterior restorations which interfere with anterior guidance (see Fig. 1). To ensure a predictable outcome it is important either to conserve anterior guidance or reorganise it in a controlled and predictable way. For work involving more than just a couple of indirect restorations access to a facebow and a lab which uses a semi-adjustable articulator is invaluable.

Problems with anterior teeth may also result from occlusal instability of the posterior teeth. The tendency for anterior teeth to splay when there is a lack of posterior teeth is easy enough to understand. Less easy is the effect of an 'anterior thrust' resulting from a deflective contact. Amar Naru has created some superb animations ${ }^{1}$ to illustrate this and other occlusal problems such as the last tooth in the arch syndrome where occlusal space mysteriously disappears after tooth preparation.

The potential for pitfalls is compounded when reorganizing a patient's occlusion, for example when restoring a worn dentition at an increased vertical dimension. Such work needs to be carefully planned and executed. Rules of thumb are useful but not always appropriate and examples will be given in the lecture. It is also important to remember that if the wear was caused by bruxism the challenges are greater in terms of maintaining occlusal control and establishing occlusal stability. Clearly, the stakes are even higher when restorations are supported by implants and great care needs to be taken to ensure the implants are not overloaded particularly when the occlusion is shared with natural teeth.

\section{Pitfalls with occlusal adjustment and splints}

Occlusal adjustment carries its own set of pitfalls. This process of reshaping the occlusal surfaces (usually with a handpiece) may be carried out before reorganising a patient's occlusion or as a means 


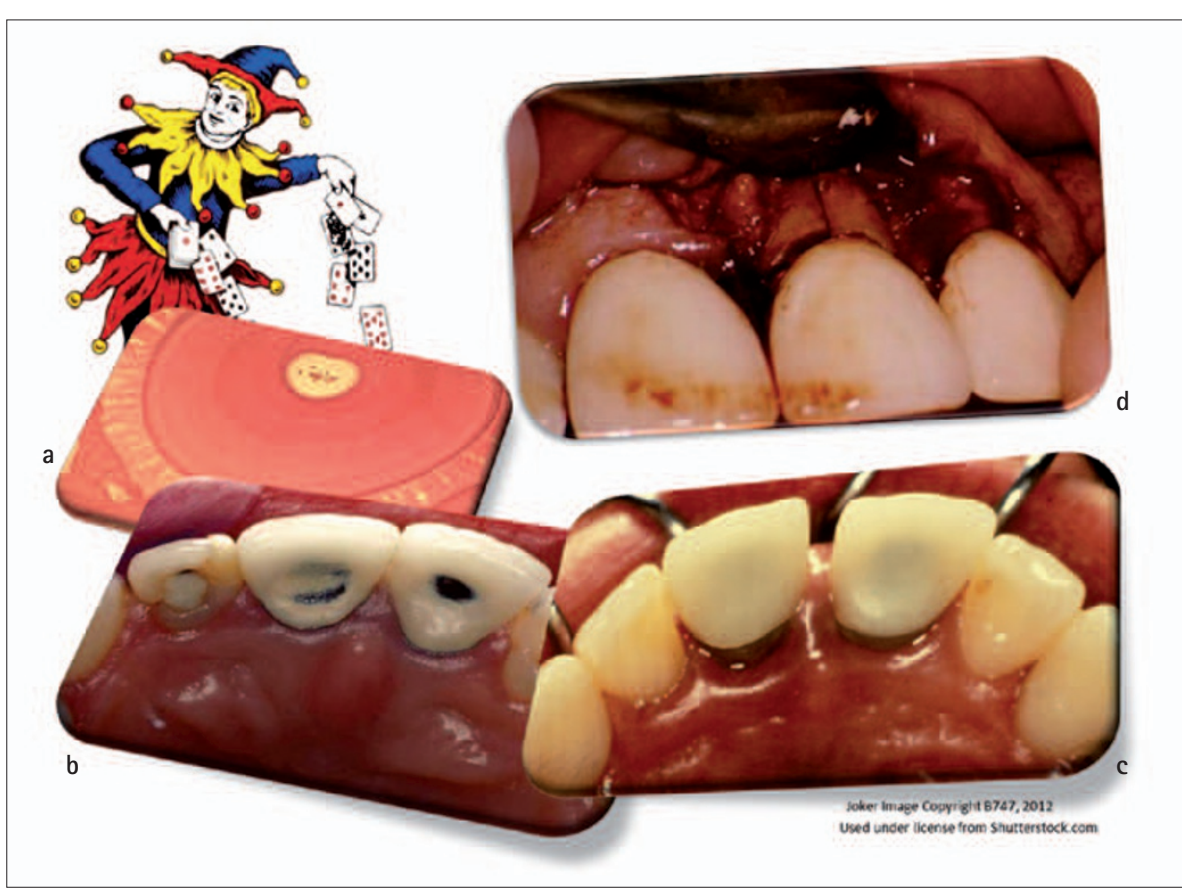

Fig. 1 It's no joke when a patient is given a crown with the wrong anterior guidance: (a) Pain may arise pulpally or from the periodontium. (b) Attempts at adjustment may leave rough ceramic which abrades opposing teeth. (c) Teeth may drift, particularly where there is periodontal disease or attachment loss. (d) Post crowns may be dislodged or roots may fracture

of optimising occlusal loading in patients with functional occlusal problems. An unfortunate pitfall occurs when dentists mislead their patients and themselves into believing that this irreversible procedure will be effective in eliminating pain associated with TMD and other chronic pain conditions (for example, idiopathic facial/ odontogenic pain). When the adjustment doesn't work or makes the pain worse the patient understandably becomes aggrieved. The situation is compounded if the dentist persists in simply adjusting teeth. To avoid such problems it is better to treat conservatively with explanation, occlusal splints and jaw exercises. Where this does not work there is a need to refer appropriately. Remember, even after splint treatment very few TMD patients actually need any occlusal adjustment.

Occlusal splints are a growth area for some dentists who risk the pitfall of falling for the splint manufacturers' hype. Nevertheless, where splints are indicated there are some subtleties of technique that can really help with getting them to fit properly.

\section{Pitfalls with hypervigilant patients}

Occlusal hypervigilance can take a number of forms. Some patients become exquisitely sensitive about the way their teeth meet to the extent that they are better at detecting interferences than many dentists. These patients appear to have an amplified level of sensation which can be troublesome when perceived as pain. Nevertheless, unlike the princess and the pea, there is often a heavy occlusal contact present - you just need to know how to look for it and adjust it. A pitfall of not recognising such a patient is unnecessary root canal treatment.

Patients who have 'phantom bite' are also hypervigilant over the way their teeth meet, but these patients do not respond well to occlusal adjustment. Indeed it can be difficult to match the patient's symptoms to the findings of the occlusal examination. These patients often travel in vain from one dentist to another in search of a solution. The unwary dentist may fall into the trap of adjusting the patient's teeth but after an initial improvement the condition worsens as does the patient's hostility.

Clearly, there are many occlusal pitfalls. Only by knowing what they are can we take action to avoid them.

1. Wassell R, Naru A, Steele J, Nohl F. Applied occlusion (Book/DVD set). p 178. London: Quintessence Publishing Co. Ltd, 2008. 